\title{
Real-world outcomes among US Merkel cell carcinoma patients initiating immune checkpoint inhibitors or chemotherapy
}

\author{
Sunandana Chandra1 (iD), Ying Zheng*,2, Shivani Pandya ${ }^{\ddagger}{ }^{\star 3}$, Ting $\mathrm{Yu}^{2}$, Mairead Kearney ${ }^{4}$, Li \\ Wang $^{\ddagger, 3}$, Ruth Kim ${ }^{5}$ \& Hemant Phatak ${ }^{\ddagger} 2$ \\ ${ }^{1}$ Northwestern Medicine, Chicago, IL 60611, USA \\ ${ }^{2}$ EMD Serono Inc., Rockland, MA 02370, USA (A business of Merck KGaA, 64293 Darmstadt, Germany) \\ ${ }^{3}$ STATinMED Research, Plano, TX 75024, USA \\ ${ }^{4}$ Merck KGaA, 64293 Darmstadt, Germany \\ ${ }^{5}$ Pfizer Inc., New York, NY 10017, USA \\ *Author for correspondence: ying.zheng@emdserono.com \\ ${ }^{\ddagger}$ Affiliation when the research was conducted
}

\begin{abstract}
Aim: Retrospectively assessed treatment patterns and clinical and economic outcomes in Merkel cell carcinoma (MCC) patients receiving recommended first-line regimens. Materials \& methods: MCC patients newly treated with either immune checkpoint inhibitors (ICls) or chemotherapies (CTs) were selected from the Veterans Health Administration database (2013-2018); 74 patients (ICls: 20 and CTs: 54) were selected. Results: Median duration of therapy was 300 days for ICls and 91 days for CTs. Time to next treatment was 245 and 184 days, respectively. Mean total (per patient per month) costs were $\$ 15,306$ (ICls) and $\$ 10,957$ (CTs), of which $51 \%$ and $86 \%$, respectively, were non-MCC therapy-related costs. Conclusion: Despite higher costs, utilization of ICls in first-line MCC shows clinical advantages over CTs in the real world.
\end{abstract}

First draft submitted: 7 May 2020; Accepted for publication: 30 July 2020; Published online:

4 September 2020

Keywords: duration of treatment $\bullet$ health care resource utilization $\bullet$ immune checkpoint inhibitors $\bullet$ Merkel cell carcinoma $\bullet$ recommended chemotherapy $\bullet$ survival $\bullet$ time to next treatment $\bullet$ Veterans Health Administration

Merkel cell carcinoma (MCC) is a rare and aggressive skin malignancy with a higher prevalence in elderly and immunocompromised populations [1-6]. MCC is currently observed in approximately 2500 people per annum in the USA; however, due to the growing elderly population, MCC incidence is predicted to reach 3284 cases in $2025[1,6,7]$. From 2000 to 2013, the incidence rate rose from 0.5 to 0.7 per 100,000 persons [7]. Per data from the Surveillance, Epidemiology and End Results program, mortality rates increased from 0.03 to 0.43 per 100,000 persons, from 1986 to 2011 in the USA [8]. In addition to age, other common risk factors associated with MCC include male sex, light skin, Merkel cell poliomavirus infection (present in $\sim 80 \%$ of MCC tumors), exposure to ultraviolet radiation and immunosuppression [9-14]. MCC is associated with poorer prognoses and higher recurrence and mortality rates compared with other skin cancers $[6,8,15]$.

Treatment choice for MCC depends on the tumor characteristics, such as the location of the tumor, advancement of the tumor, regional lymph node involvement as well as the comorbidities and performance status of the patient [1618]. Generally, the standard treatment procedures for local tumors are surgery and radiotherapy, accompanied by chemotherapy (CT) $[19,20]$, although the use of adjuvant chemotherapy in the treatment of local tumors is clinically rare [21]. Surgery followed by radiotherapy for local tumors is associated with improved local and regional relapsefree survival and distant metastasis-free survival, as compared with surgery alone [22]. Metastatic MCC (mMCC) is also sensitive to CT but is associated with limited response durability and high toxicity, underscoring the need for more effective treatment options $[1,19,23]$. Previous retrospective studies have reported the response rate for mMCC patients treated with CT to be $52-61 \%$ in the first-line (1L) setting, and about $23 \%$ in the second-line $(2 \mathrm{~L})$ setting, with response duration ranging from 3 to 10 months [24-26].

Future Medicine 
The advent of immune checkpoint inhibitors (ICIs) has broadened the scope of treatment options for patients with mMCC [27-29]. The 2019 National Comprehensive Cancer Network (NCCN) guidelines reflect the recommendation to treat mMCC with ICIs if a clinical trial is unavailable - particularly with avelumab, pembrolizumab and nivolumab [19]. Furthermore, avelumab, a PD-L1 monoclonal antibody, was the first ICI, and therapy overall, approved by the US FDA in March 2017 for the treatment of mMCC among adults and pediatric patients 12 years and older [17]. The approval was based on results from the JAVELIN Merkel 200 Phase II clinical trial in which 32\% of 88 patients with mMCC who were previously treated with CT responded to the drug, with $9 \%$ experiencing complete response [30]. At the time of data cutoff, the median duration of response (range: at least 2.8 months to at least 17.5 months) was not reached [27]. A follow-up analyses conducted among patients with at least 1 year of follow-up by Kaufman et al. concluded that avelumab treatment results in durable efficacy and prolonged progression-free survival (PFS) [31]. Although median PFS was 2.7 months, the Kaplan-Meier (KM)-estimated PFS curve for avelumab reached a plateau, which suggests long-term clinical benefits [31]. In December 2018, the FDA granted approval to a second checkpoint blockade therapy, pembrolizumab, anti-PD-1 monoclonal antibody [26]. The approval was granted based on the results of the Cancer Immunotherapy Trials Network protocol 9/Keynote017 Phase II trial in which $56 \%$ of 50 treatment native patients with locally advanced or mMCC responded to the drug, with $24 \%$ experiencing complete remission [32]. Median response duration (range: at least 5.9 months to longer than 34.5 months) was not achieved [32]. It should be noted that other ICIs (e.g., nivolumab and ipilimumab) are currently being evaluated in clinical trials to determine their potential use in mMCC management [33].

A weakened immune system is an established risk factor for MCC. More specifically, T-cell dysfunction is associated with a dramatically higher incidence of the malignancy [34]; for example, patients with HIV and solid organ transplants are 11-13-times and 5-10-times more likely to develop MCC, respectively, than the general population [35]. In addition, the role of Merkel cell poliomavirus in the pathogenesis of MCC depends on the presence of additional immunosuppression from UV radiation, environmental mutagens, old age, infections or drugs. Furthermore, the link between MCC survival and the immune system has been previously studied; agents which promote an antitumor immune response may increase the chances of survival [36-39]. However, studies which explore the characteristics of patients with MCC and a compromised immune system are lacking.

While some real-world data studies have examined the economic burden and treatment patterns associated with MCC in the USA [19,40,41], comprehensive studies on these topics inclusive of newly available ICI therapies are sparse. Veterans are at a particularly high risk of skin malignancies, including MCC, due to occupational sun exposure for extended periods of time [42,43]; a previous study among the veteran population identified 346 MCC patients between 1995 and 2006 [44]. In addition, the US Veterans Health Administration (VHA) database is made up of mostly elderly and male patients [45], which constitutes a high-risk group for MCC. However, there is a lack of literature evaluating treatment patterns and economic outcomes for MCC among veterans or within the VHA. This study sought to evaluate treatment patterns, clinical outcomes and economic outcomes (healthcare resource utilization [HCRU] and costs) among MCC patients treated with CTs or ICIs using the US VHA database. In addition, the characteristics of MCC patients with and without compromised immune systems were examined noncomparatively.

\section{Materials \& methods \\ Data source}

This retrospective observational claims-based study analyzed the medical and pharmacy service records from the VHA database, from 1 April 2013 through 31 March 2018. The VHA is the largest integrated healthcare system in the USA. It provides veterans a broad spectrum of healthcare services (e.g., inpatient, outpatient and long-term) and captures information for over 9 million veterans across the country [45]. The study data were extracted from VHA Medical Inpatient and Outpatient Medical SAS datasets and from VHA Decision Support System datasets. The VHA Decision Support System is a longitudinal, relational database combining the clinical and cost data needed to integrate expenses and utilization of various services and allocate the costs to appropriate services provided to veterans [46].

Diagnostic information was identified using International Classification of Disease, Ninth Revision, Clinical Modification (ICD-9-CM/ICD-10-CM) codes on the inpatient and outpatient claims. Systemic therapies were identified using the relevant Health Care Common Procedure Coding System (HCPCS) codes and National Drug Codes (NDC). The VHA vital status file provides information on the demographics and the vital status of each patient. Since the core study proposed herein did not involve the collection, use or transmittal of individual 
identifiable data, Institutional Review Board (IRB) approval to conduct this study was not required as per VHA's classification of de-identified data for release.

\section{Patient selection \& identification}

The study included patients diagnosed with MCC (ICD-9-CM: 209.31-209.36, 209.75; ICD-10-CM: C4A, C7B.1) who subsequently had evidence of any MCC systemic therapy during the study identification period from 1 October 2013 through 31 January 2018. The first MCC diagnosis date during the identification period was defined as the initial diagnosis date. The first systemic therapy date after initial diagnosis date was defined as the index date. In addition, patients were required to have continuous medical and pharmacy enrollment for $\geq 6$ months pre-initial MCC diagnosis date until $\geq 2$ months post-index date, no evidence of MCC diagnosis or systemic therapy during the 6 months prior to the initial diagnosis date and index date, respectively. Patient follow-up period ranged until the earliest of health plan disenrollment, death or end of the study period. The 6-month period prior to the index date was defined as the baseline period. Our study primarily focused on patients treated with NCCN-recommended regimens for metastatic disease [20], including ICIs or CT regimens; this was used as a proxy to select for patients with advanced or mMCC. The patients were assigned to two mutually exclusive cohorts: the ICI and the CT cohorts based on the treatment received on the index date. For treatment cycles that started with a combination of ICI and CT on the index date, ICI therapy was given priority and the cycle length was based on the ICI therapy observed on the index date.

In parallel, patients were categorized as immunocompromised and nonimmunocompromised. Patients were included into the immunocompromised cohort if they had any of the following conditions in the baseline period: T-cell count $<0.5 \mathrm{~K} / \mathrm{mm}^{3}$, diagnosis of HIV, select hematologic diseases (chronic lymphocytic leukemia, multiple myeloma and hypogammaglobulinemia), organ transplant, allogeneic stem cell transplant or evidence of select immunosuppressive treatment. Patients without evidence of these conditions were included in the nonimmunocompromised cohort.

\section{Study measures}

Patient characteristics

Baseline characteristics were examined for all patients and included demographics such as age on the index date, sex and race. The comorbidity burden during the baseline period was evaluated using the Quan-Charlson Comorbidity Index (CCI) which assigns weights to 17 comorbidities such as liver disease, diabetes, cancer and many more. These weights are then added together to create a summary indicator of baseline health status. A higher CCI score indicates greater baseline risk [47,48]. In addition, certain individual comorbidities, time (in days) from initial diagnosis date until index date, all-cause and MCC-related HCRU and healthcare costs during the baseline period were also evaluated.

\section{Treatment duration}

The lines of therapy were determined using a claims data algorithm based on the start date and end date of a treatment regimen. The treatment lines were defined in two steps. First, treatment cycles were identified. The first claim identified for any therapy on the index date marked the start of the first cycle of $1 \mathrm{~L}$ therapy. The cycle length was set at 14 or 21 days. Cycles starting with ICIs (e.g., avelumab or nivolumab) or a combination of ICIs and CT on the index date ended after 14 days; cycles starting with CTs or pembrolizumab on the index date ended after 21 days. The $1 \mathrm{~L}$ regimen was composed of all systemic therapies observed within the first cycle. Consecutive cycles consisting of the same therapies in the $1 \mathrm{~L}$ regimen were regarded as continuations of $1 \mathrm{~L}$ if the gap between the cycles did not exceed 60 days. When the gap between cycles exceeded 60 days, or when the existing regimen was changed by the addition of a new therapy or a switch from all therapies in the regimen, a new line of therapy was considered to begin (Figure 1). Duration of $1 \mathrm{~L}$ therapy was measured as the period (in days) from the index date until the earliest of day of the last cycle before the start of a new treatment line, or follow-up end.

\section{Clinical outcomes}

The clinical outcomes evaluated in this study included time to next treatment (TTNT) and time to death. TTNT was defined as the time (in days) from the index date to the earliest occurrence of initiation of $2 \mathrm{~L}$ therapy or death due to any cause. Time to death was defined as the time (in days) from the index date to the date of death, due to any cause. KM curves were used to evaluate the median TTNT and time to death. 


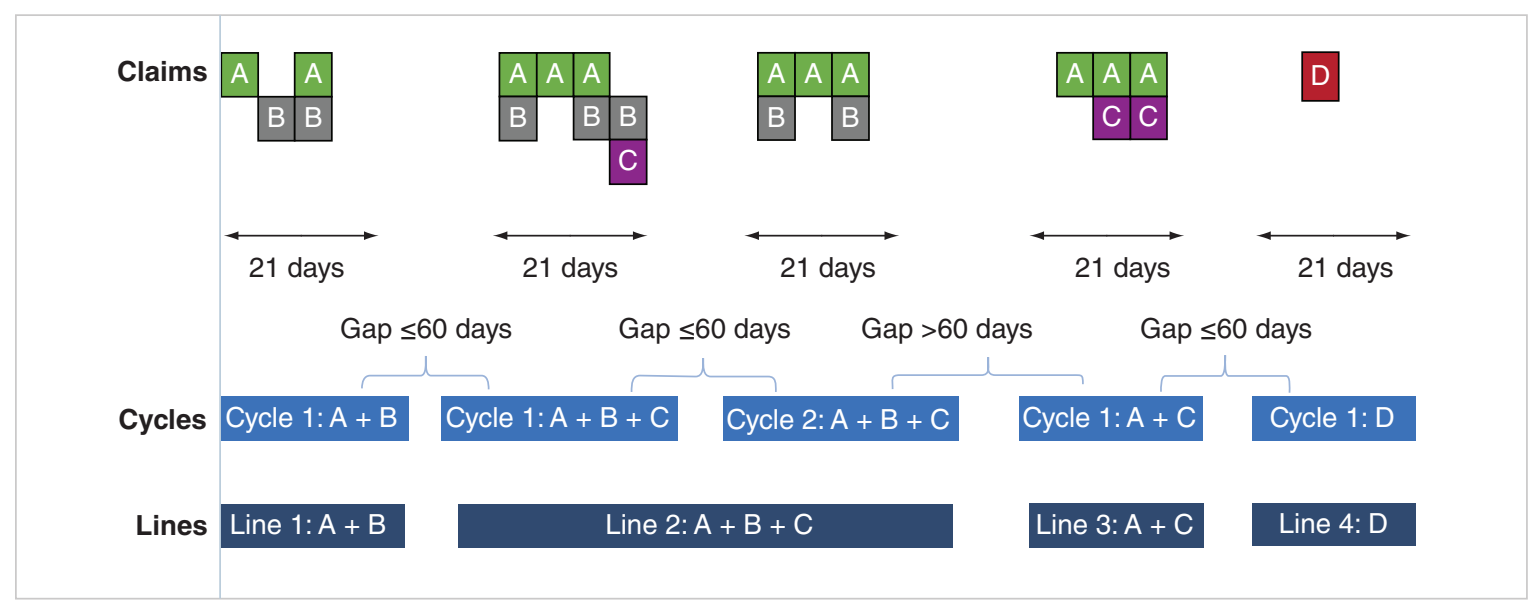

Figure 1. Schematic representation of treatment patterns. A-D indicate distinct systemic therapies. Gap is calculated from the end of a cycle until the start of the next cycle or follow-up end.

\section{Economic outcomes}

All-cause HCRU and direct healthcare costs were reported from index date until next treatment (i.e., for the duration of $1 \mathrm{~L}$ therapy and any treatment-free interval before the earliest of start of $2 \mathrm{~L}$ or death). Direct healthcare costs were evaluated from a payer perspective and covered cost for all care received in the inpatient, outpatient and pharmacy settings. Inpatient visit (including length of stay) and expenditures included the care received in the acute care and extended care settings (e.g., nursing homes). Outpatient visits and expenditures included the care received in any outpatient setting, including outpatient office, emergency department visits, laboratory services and all other services. Pharmacy visits and expenditures included prescriptions from inpatient and outpatient pharmacy. Healthcare resource use and costs were reported in total as well as for the following subcategories: MCC therapy related (including outpatient encounters on a day when systemic therapy was administered and pharmacy encounters for systemic therapy) and non-MCC therapy related (including inpatient encounters, outpatient encounters on a day without any evidence of systemic therapy administration, and pharmacy encounters for non-MCC therapies).

\section{Statistical analysis}

All study variables, including patient demographic and clinical characteristics as well as the study outcomes were described using standard descriptive statistics among patients in the ICI and CT cohorts. Categorical variables were summarized in the form of numbers and percentages. Continuous variables were summarized in the form of mean and standard deviation (SD). Additionally, descriptive analyses were also conducted to examine the baseline demographic and clinical characteristics of patients in the immunocompromised versus nonimmunocompromised cohorts.

KM survival analysis was conducted to estimate the median duration of $1 \mathrm{~L}$ therapy, TTNT and time to death by the $1 \mathrm{~L}$ treatment type; 95\% CIs were also estimated from KM analysis. Given the variable TTNT for each patient, HCRU and costs were reported on a per patient per month (PPPM) basis, by dividing each patients' total visits and costs accrued during TTNT by the duration of TTNT (in months) and calculating the group average for all of the individual patient averages. The costs were adjusted to 2018 US dollars using the medical care component of the Consumer Price Index (CPI) from the US Department of Labor [49].

\section{Results}

A total of 74 patients with incident MCC receiving treatment with NCCN-recommended $1 \mathrm{~L}$ regimens qualified for the study. This study-qualified population was segmented into a cohort of $20(27.0 \%)$ patients initiating ICIs and a cohort of $54(73.0 \%)$ patients initiating CTs (Figure 2).

\section{Demographic \& baseline clinical characteristics for the CT \& ICl cohorts}

Patients with MCC receiving 1L systemic therapy in both cohorts were predominantly White $(87.8 \% ; \mathrm{n}=65)$ and male $(98.6 \% ; n=73)$. The mean $( \pm S D)$ age ranged from $72.4 \pm 6.6$ years in the ICI cohort to $69.2 \pm 7.7$ years 


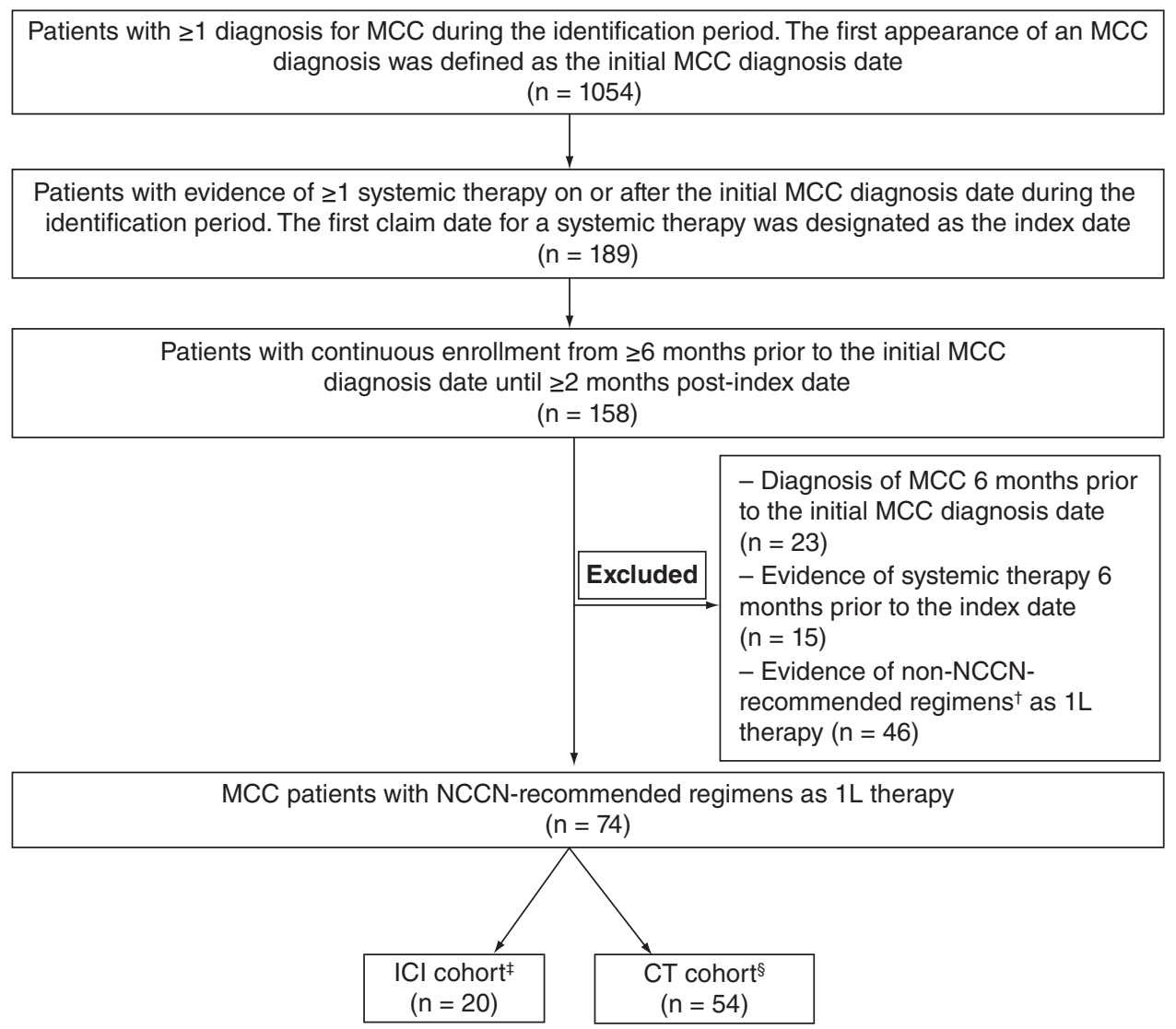

Figure 2. Patient selection criteria.

†Non-NCCN-recommended regimens include fluorouracil; docetaxel; carboplatin; carboplatin + paclitaxel; cisplatin; etoposide; gemcitabine; irinotecan; carboplatin + gemcitabine; cyclophosphamide + doxorubicin + vincristine; and pembrolizumab + carboplatin + etoposide.

$¥ \mathrm{NCCN}$-recommended ICI therapies include avelumab; pembrolizumab or nivolumab.

$\S$ NCCN-recommended CT regimens include cisplatin \pm etoposide; carboplatin \pm etoposide; topotecan; cyclophosphamide; doxorubicin (or epirubicin) and vincristine (CAV).

1L: First-line; CT: Chemotherapy; ICl: Immune checkpoint inhibitor; MCC: Merkel cell carcinoma; NCCN: National Comprehensive Cancer Network.

in the CT cohort (Table 1). In both cohorts, most patients were aged $\geq 65$ years (ICI cohort: $95 \% ; n=19$; CT cohort: $77.8 \% ; \mathrm{n}=42$ ), male (ICI cohort: $95 \% ; \mathrm{n}=19$; CT cohort: $100 \% ; \mathrm{n}=54$ ), and White (ICI cohort: $85 \% ; \mathrm{n}=17$; CT cohort: $88.9 \% ; \mathrm{n}=48$ ).

Both cohorts had substantial comorbidity as indicated by mean $( \pm S D)$ Quan-CCI scores (ICI cohort: $6.8 \pm 4.7$; CT cohort: $6.4 \pm 4.0$ ) and rates of individual comorbidities (Table 1). The mean time from MCC diagnosis to index date was $200 \pm 164$ days in the ICI cohort and $110 \pm 125$ days in the CT cohort. Baseline mean all-cause PPPM inpatient, outpatient and pharmacy visits for the ICI cohort were $0.19 \pm 0.29,4.58 \pm 2.94$ and $3.92 \pm 2.62$ visits, respectively; for the CT cohort, there were $0.15 \pm 0.27,4.13 \pm 2.44$ and $3.86 \pm 3.09$ mean all-cause PPPM inpatient, outpatient and pharmacy visits, respectively. Baseline mean MCC-related PPPM inpatient and outpatient visits for the ICI cohort were $0.13 \pm 0.16$ and $1.46 \pm 1.79$ visits, respectively; for the CT cohort, there were $0.06 \pm 0.12$ and $1.02 \pm 1.21$ mean MCC-related PPPM inpatient and outpatient visits, respectively.

Baseline mean all-cause PPPM medical healthcare costs were $\$ 7637 \pm \$ 6170$ for the ICI and $\$ 7258 \pm \$ 8957$ for the CT cohort. Of these, $\$ 4409 \pm \$ 5049$ (ICI cohort) and $\$ 2605 \pm \$ 3482$ (CT cohort) were MCC related. Baseline mean all-cause PPPM total healthcare costs were $\$ 8464 \pm \$ 6200$ and $\$ 7654 \pm \$ 9246$ for the ICI and CT cohorts, respectively. 
Table 1. Baseline demographic and clinical characteristics of Merkel cell carcinoma patients prescribed recommended first-line systemic therapy, stratified by treatment type.

Demographic and clinical characteristics MCC patients with 1 L systemic therapy $(n=74)$

\begin{tabular}{|c|c|c|}
\hline & \\
\hline & ICI cohort $(n=20)$ & CT cohort $(n=54)$ \\
\hline Age (years; mean $\pm S D$, median [range]) & $72.4 \pm 6.6,70.5(64.0-84.0)$ & $69.2 \pm 7.7,68.5(50.0-85.0)$ \\
\hline \multicolumn{3}{|l|}{ Age category $(n, \%)$} \\
\hline$-\geq 65$ years & $19(95.0 \%)$ & $42(77.8 \%)$ \\
\hline \multicolumn{3}{|l|}{ Gender (n, \%) } \\
\hline - Male & $19(95.0 \%)$ & $54(100.0 \%)$ \\
\hline \multicolumn{3}{|l|}{ Race $(n, \%)$} \\
\hline - White & $17(85.0 \%)$ & $48(88.9 \%)$ \\
\hline \multicolumn{3}{|l|}{ Baseline clinical characteristics } \\
\hline - Quan-CCI score (mean \pm SD, median [range]) & $6.8 \pm 4.7,8.0(0.0-15.0)$ & $6.4 \pm 4.0,7.0(0.0-15.0)$ \\
\hline \multicolumn{3}{|l|}{ Individual comorbidities (n, \%) } \\
\hline \multicolumn{3}{|l|}{$\mathrm{CCl}$ conditions } \\
\hline - Any (non-MCC) malignancy & $13(65.0 \%)$ & $28(51.9 \%)$ \\
\hline - Metastatic (non-MCC) solid tumor & $13(65.0 \%)$ & $33(61.1 \%)$ \\
\hline - Other & $12(60.0 \%)$ & $37(68.5 \%)$ \\
\hline - Time from MCC date to index date (days; mean \pm SD, median [range]) & $200 \pm 164,151(2-493)$ & $110 \pm 125,49(0-538)$ \\
\hline \multicolumn{3}{|l|}{ Baseline economic measures (mean $\pm \mathrm{SD}$, median [range]) } \\
\hline \multicolumn{3}{|l|}{ Baseline all-cause HCRU PPPM } \\
\hline - Inpatient visits & $0.19 \pm 0.29,0.17(0.00-1.33)$ & $0.15 \pm 0.27,0(0.00-1.67)$ \\
\hline - Outpatient visits & $4.58 \pm 2.94,3.92(0.50-12.83)$ & $4.13 \pm 2.44,3.67(0.67-11.00)$ \\
\hline - Pharmacy visits & $3.92 \pm 2.62,3.08(0.50-9.67)$ & $3.86 \pm 3.09,3.25(0.00-15.00)$ \\
\hline \multicolumn{3}{|l|}{ Baseline MCC-related HCRU PPPM } \\
\hline - Inpatient visits & $0.13 \pm 0.16,0.17(0.00-0.67)$ & $0.06 \pm 0.12,0(0.00-0.50)$ \\
\hline - Outpatient visits & $1.46 \pm 1.79,0.83(0.00-6.50)$ & $1.02 \pm 1.21,0.67(0.00-7.00)$ \\
\hline \multicolumn{3}{|l|}{ Baseline all-cause healthcare costs PPPM } \\
\hline - Inpatient costs & $\$ 4011 \pm \$ 4744, \$ 2121(\$ 0-\$ 15,056)$ & $\$ 3559 \pm \$ 7416, \$ 0(\$ 0-\$ 36,296)$ \\
\hline - Outpatient costs & $\$ 3626 \pm \$ 2917, \$ 2923(\$ 366-\$ 11,267)$ & $\$ 3699 \pm \$ 2685, \$ 3058(\$ 310-\$ 13,799)$ \\
\hline - Pharmacy costs & $\$ 827 \pm \$ 1732, \$ 344(\$ 18-\$ 7836)$ & $\$ 396 \pm \$ 732, \$ 133(\$ 0-\$ 4495)$ \\
\hline - Total medical costs (outpatient + inpatient) & $\$ 7637 \pm \$ 6170, \$ 4456(\$ 1061-\$ 19,284)$ & $\$ 7258 \pm \$ 8957, \$ 3651(\$ 310-\$ 46,782)$ \\
\hline - Total costs (outpatient + inpatient + pharmacy) & $\$ 8464 \pm \$ 6200, \$ 5903(\$ 1277-\$ 19,616)$ & $\$ 7654 \pm \$ 9246, \$ 3774(\$ 319-\$ 47,580)$ \\
\hline \multicolumn{3}{|l|}{ Baseline MCC-related healthcare costs PPPM } \\
\hline - Inpatient costs & $\$ 2859 \pm \$ 4236, \$ 1677(\$ 0-\$ 15,056)$ & $\$ 1340 \pm \$ 3137, \$ 0(\$ 0-\$ 13,846)$ \\
\hline - Outpatient costs & $\$ 1550 \pm \$ 2099, \$ 660(\$ 0-\$ 6709)$ & $\$ 1265 \pm \$ 1749, \$ 748(\$ 0-\$ 10,339)$ \\
\hline - Total medical costs (outpatient + inpatient) & $\$ 4409 \pm \$ 5049, \$ 2472(\$ 0-\$ 15,056)$ & $\$ 2605 \pm \$ 3482, \$ 1199(\$ 0-\$ 14,930)$ \\
\hline
\end{tabular}

Treatment duration

The median duration of $1 \mathrm{~L}$ therapy was 300 days (95\% CI: 91-364 days) for the ICI cohort and 91 days (95\% CI: 63-105 days) for the CT cohort (Figure 3). In addition, 90.0\% ( $n=18)$ of the ICI cohort and $81.5 \%(n=44)$ of the CT cohort had received at least 1 month's treatment; $75.0 \%(n=15)$ and $59.3 \%(n=32)$, respectively, had at least 2 months' treatment.

\section{Clinical outcomes}

The median TTNTs for the ICI and CT cohorts were 245 (95\% CI: 124 days - upper limit not reached) and 184 days (95\% CI: 127-324 days), respectively (Figure 4).

While the median time to death was not achieved for the ICI cohort (95\% CI: 132 days - upper limit not reached), the median time to death was 403 days (95\% CI: 280-630 days) for the CT cohort (Figure 5). 


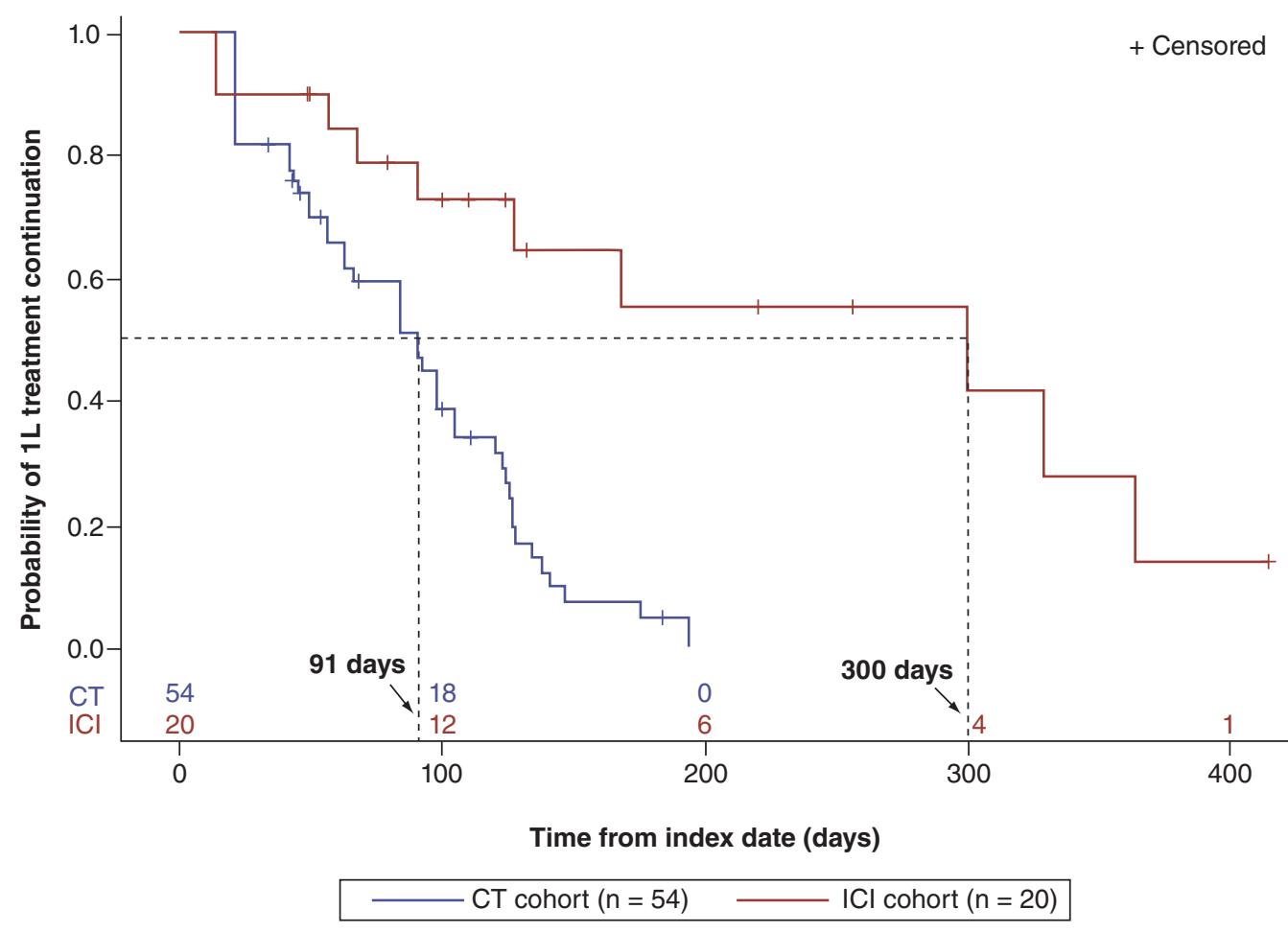

Figure 3. Kaplan-Meier curves for duration of therapy among Merkel cell carcinoma patients prescribed recommended first-line systemic therapy stratified by treatment type.

1L: First line; CT: Chemotherapy; ICI: Immune checkpoint inhibitor.

\section{Economic outcomes}

During TTNT, the mean MCC therapy-related PPPM medical visits were $1.61 \pm 0.79$ visits $(1.03 \pm 0.47$ pharmacy visits, $0.58 \pm 0.50$ outpatient visits) for the ICI cohort and $1.79 \pm 1.62$ visits $(1.16 \pm 1.08$ pharmacy visits, $0.63 \pm 0.95$ outpatient visits) for the CT cohort. Mean non-MCC therapy-related PPPM medical visits were $7.09 \pm 2.70$ visits $(3.47 \pm 1.91$ pharmacy visits, $3.41 \pm 1.79$ outpatient visits, $0.21 \pm 0.38$ inpatient visits) for the ICI cohort and $8.45 \pm 5.34$ visits $(4.57 \pm 4.05$ pharmacy visits, $3.71 \pm 2.42$ outpatient visits, $0.17 \pm 0.25$ inpatient visits) for the CT cohort (Figure 6). The mean all-cause PPPM length of inpatient stay was $0.94 \pm 2.13$ days for the ICI cohort and $1.39 \pm 3.08$ days for the CT cohort.

Total mean all-cause PPPM costs were $\$ 15,306 \pm \$ 13,833$ for the ICI cohort and $\$ 10,957 \pm \$ 13,826$ for the CT cohort during TTNT. Costs in the ICI cohort were driven mainly by outpatient care (\$7529 $\pm \$ 7244)$ followed by pharmacy $(\$ 5184 \pm \$ 5511)$, while costs in the CT cohort were driven mainly by inpatient $(\$ 4810 \pm \$ 12,514)$ care followed by outpatient $(\$ 4339 \pm \$ 3091)$. For the ICI cohort, $49.3 \%$ of costs $(\$ 7540 \pm \$ 9552)$ were due to MCC therapy-related services, driven mainly by MCC therapy-related pharmacy costs; $50.7 \%$ of costs $(\$ 7766 \pm \$ 7744)$ were due to non-MCC therapy-related services, driven mainly by non-MCC therapy-related outpatient costs. For the CT cohort, $13.5 \%$ of costs $(\$ 1482 \pm \$ 1832)$ were due to MCC therapy-related services, driven mainly by MCC therapy-related outpatient costs; $86.5 \%$ of costs $(\$ 9476 \pm \$ 13,598)$ were due to non-MCC therapy-related services (Figure 7), driven mainly by inpatient costs.

Demographic \& baseline characteristics for the immunocompromised \& nonimmunocompromised cohorts

Patients were also segmented based on their immunocompromised status: 18 (24.3\%) patients were assigned to the immunocompromised cohort, while $56(75.7 \%)$ were included in the nonimmunocompromised cohort. The mean age ranged from $70.5 \pm 8.5$ years in the immunocompromised cohort to $69.8 \pm 7.3$ years in the nonimmunocompromised cohort. In both cohorts, most patients were aged $\geq 65$ years (immunocompromised 


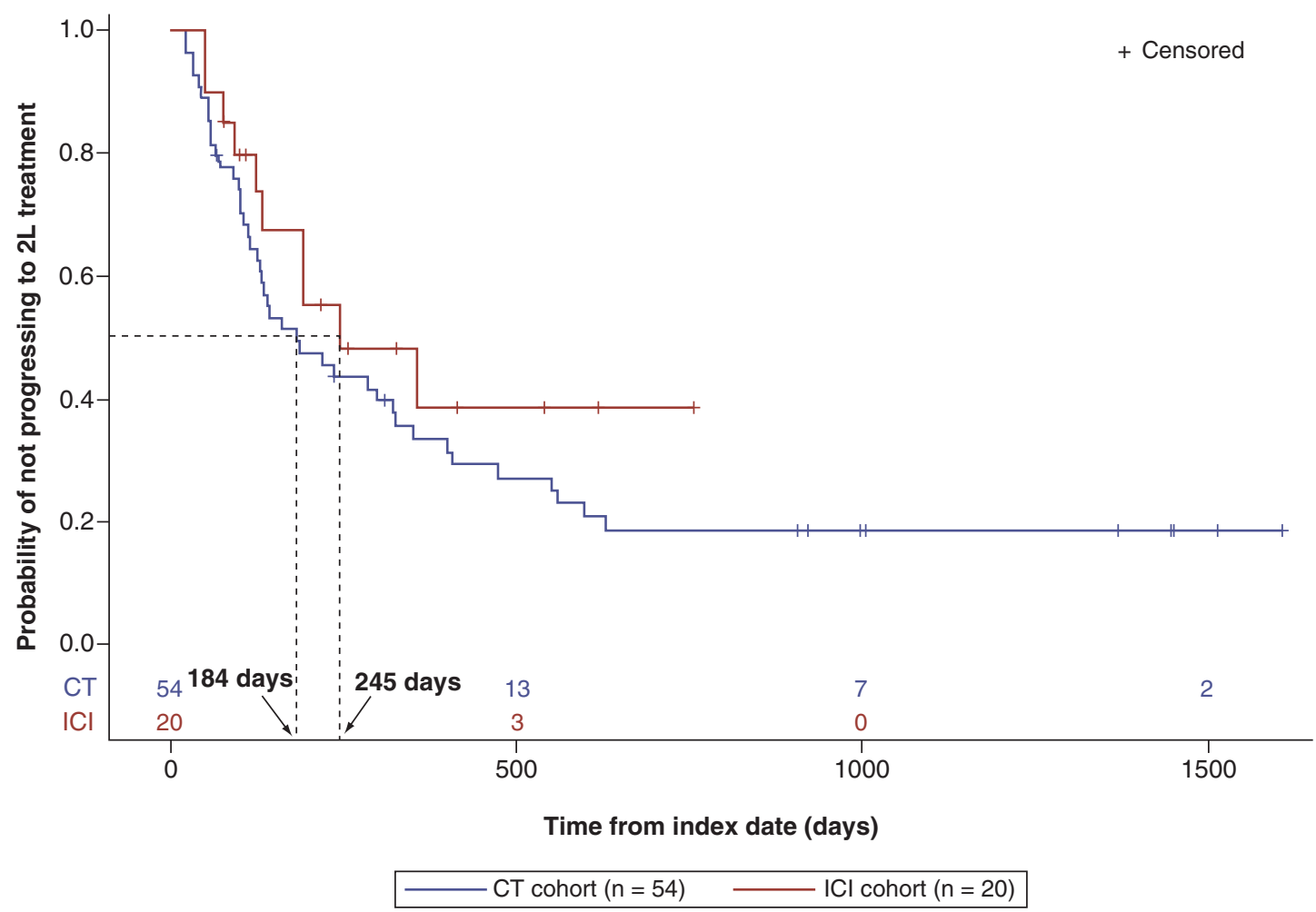

Figure 4. Kaplan-Meier curves for time to next treatment among Merkel cell carcinoma patients prescribed recommended first-line systemic therapy stratified by treatment type.

1L: First line; CT: Chemotherapy; ICI: Immune checkpoint inhibitor.

cohort: $72.2 \% ; \mathrm{n}=13$; nonimmunocompromised cohort: $85.7 \% ; \mathrm{n}=48$ ), male (immunocompromised cohort: $100 \% ; \mathrm{n}=18$; nonimmunocompromised cohort: $98.2 \% ; \mathrm{n}=55$ ) and White (immunocompromised cohort: $83.3 \% ; \mathrm{n}=15$; nonimmunocompromised cohort: $89.3 \% ; \mathrm{n}=50$ ). Both cohorts had substantial comorbidity burden with high rates of individual comorbidities including any malignancies except MCC or metastatic solid tumors (Table 2).

The mean time from an MCC diagnosis to the index date was $173 \pm 146$ days in the immunocompromised cohort and $122 \pm 139$ days in the nonimmunocompromised cohort. Baseline mean all-cause PPPM inpatient, outpatient and pharmacy visits were $0.27 \pm 0.34,5.26 \pm 2.56$ and $5.43 \pm 3.10$ visits, respectively, for the immunocompromised cohort and $0.13 \pm 0.25,3.93 \pm 2.51$ and $3.38 \pm 2.76$ visits, respectively, for the nonimmunocompromised cohort. Baseline mean MCC-related PPPM inpatient and outpatient visits were $0.15 \pm 0.18$ and $1.08 \pm 1.02$ visits, respectively, for the immunocompromised cohort and $0.06 \pm 0.11$ and $1.15 \pm 1.50$ visits, respectively, for the nonimmunocompromised cohort. Baseline mean all-cause PPPM medical costs were $\$ 9320 \pm \$ 10,626$ for the immunocompromised cohort; costs for MCC-related medical visits were $\$ 3541 \pm \$ 3357$. Baseline mean allcause PPPM medical costs were $\$ 6730 \pm \$ 7345$ for the nonimmunocompromised cohort; costs for MCC-related medical visits were $\$ 2949 \pm \$ 4217$. Baseline mean all-cause PPPM total costs were $\$ 10,104 \pm \$ 10,702$ and $\$ 7156 \pm \$ 7634$ for the immunocompromised and nonimmunocompromised cohorts, respectively.

\section{Discussion}

This noncomparative claims-based retrospective study described patient characteristics, treatment patterns and clinical and economic outcomes among newly diagnosed MCC patients in the US treated with ICI therapies or NCCN-recommended CTs as 1L systemic therapy. To our knowledge, this study represents the first detailed clinical and economic description of MCC care using the VHA database. The VHA database is an all-electronic 


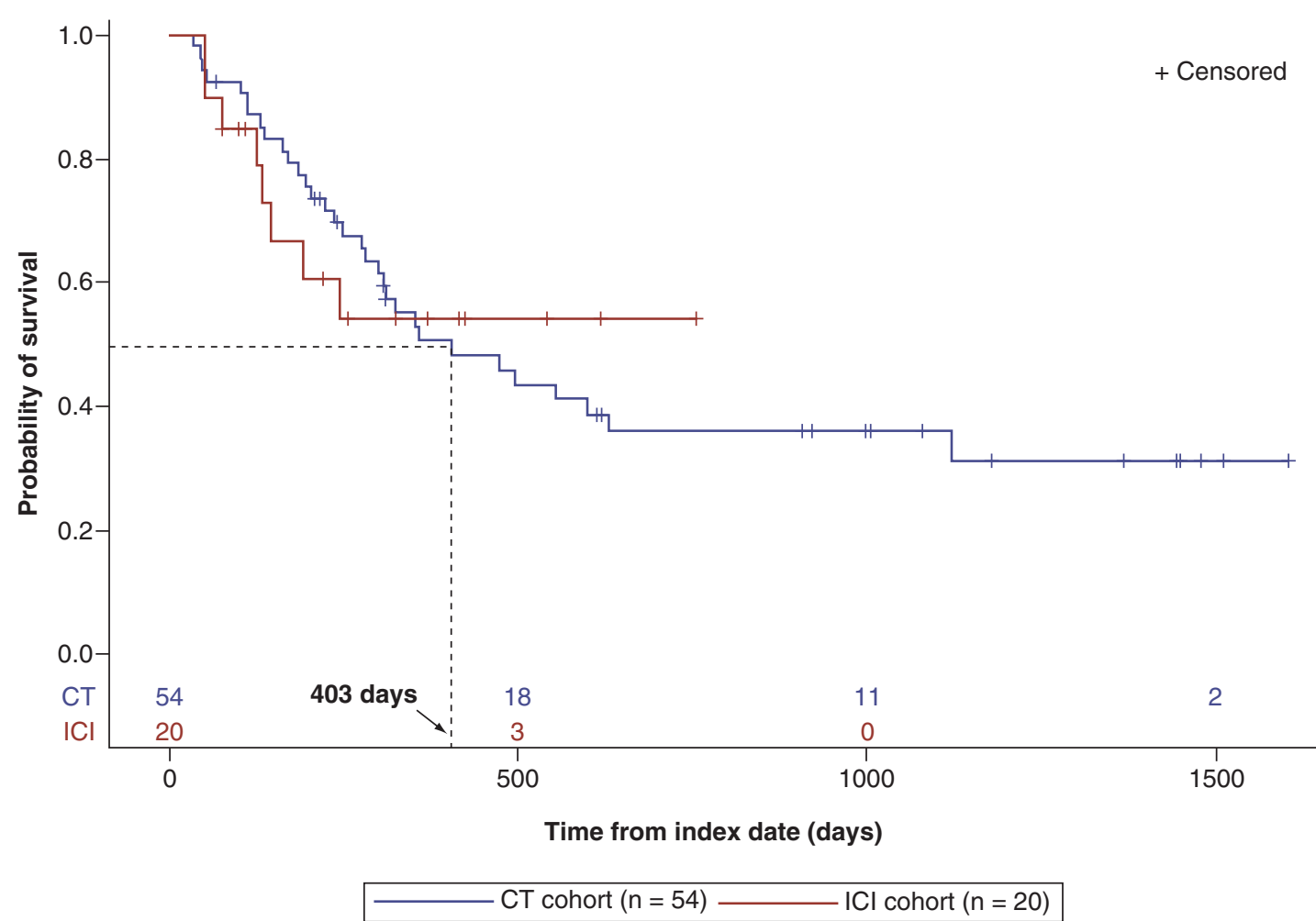

Figure 5. Kaplan-Meier curves for time to death among Merkel cell carcinoma patients prescribed recommended first-line systemic therapy stratified by treatment type.

CT: Chemotherapy; ICI: Immune checkpoint inhibitor.

health record system, which captures extensive patient care details for a large population in a fully integrated claims database. This makes the database well suited for epidemiological studies.

Our study found that, among veterans registered with the VHA, newly diagnosed and newly treated MCC patients receiving $1 \mathrm{~L}$ systemic therapy were mostly elderly, male and White. This pattern of sociodemographic characteristics among MCC patients has been previously reported [19,43]. Our study did report a much higher proportion of male patients with MCC compared with previous studies [50-52], in which male to female ratios of 6:5 or 1.5:1 have been observed among MCC patients. The excess of male MCC patients here reflects the demographic of the larger VHA population (i.e., elderly and male). In addition, MCC patients in this study had a high baseline mean $( \pm S D)$ Quan-CCI score (6.5 \pm 4.2$)$ which exceeds that previously reported $(2.1 \pm 2.3)$ [19]. The difference in scores between these studies may reflect the difference in comorbidity indices used in each study. It should also be noted that relative baseline HCRU and costs were in accordance with average comorbidity scores among treatment cohorts (i.e., higher Quan-CCI scores were correlated with higher HCRU and costs). This agrees with a previous study that infers how HCRU and costs correlate with comorbidity level [19]. However, this was a noncomparative study and further research should compare comorbidity levels and economic burden between treatment cohorts using appropriate statistical tests of significance.

Our study is also the first real-world study to examine treatment duration and clinical outcomes associated with ICIs for the $1 \mathrm{~L}$ management of MCC. Kearney et al. examined 1L treatment duration for MCC patients from 1 July 2010 to 31 December 2014 [19]. The KM curve-derived median estimate for duration of 1L CT in that study was 98 days which is similar to our findings for median duration of 1L CT (91 days) [19,41]. Our study found an extended duration of therapy (300 days) within the ICI cohort. This supports findings from previous studies that have reported an early and durable response among patients treated with avelumab and pembrolizumab [53-55]. In addition, we observed that TTNT (as measured in the current study as progression to $2 \mathrm{~L}$ or death) for ICI was 245 days, numerically higher than that for CT (184 days). Our definition of TTNT is analogous to the definition 


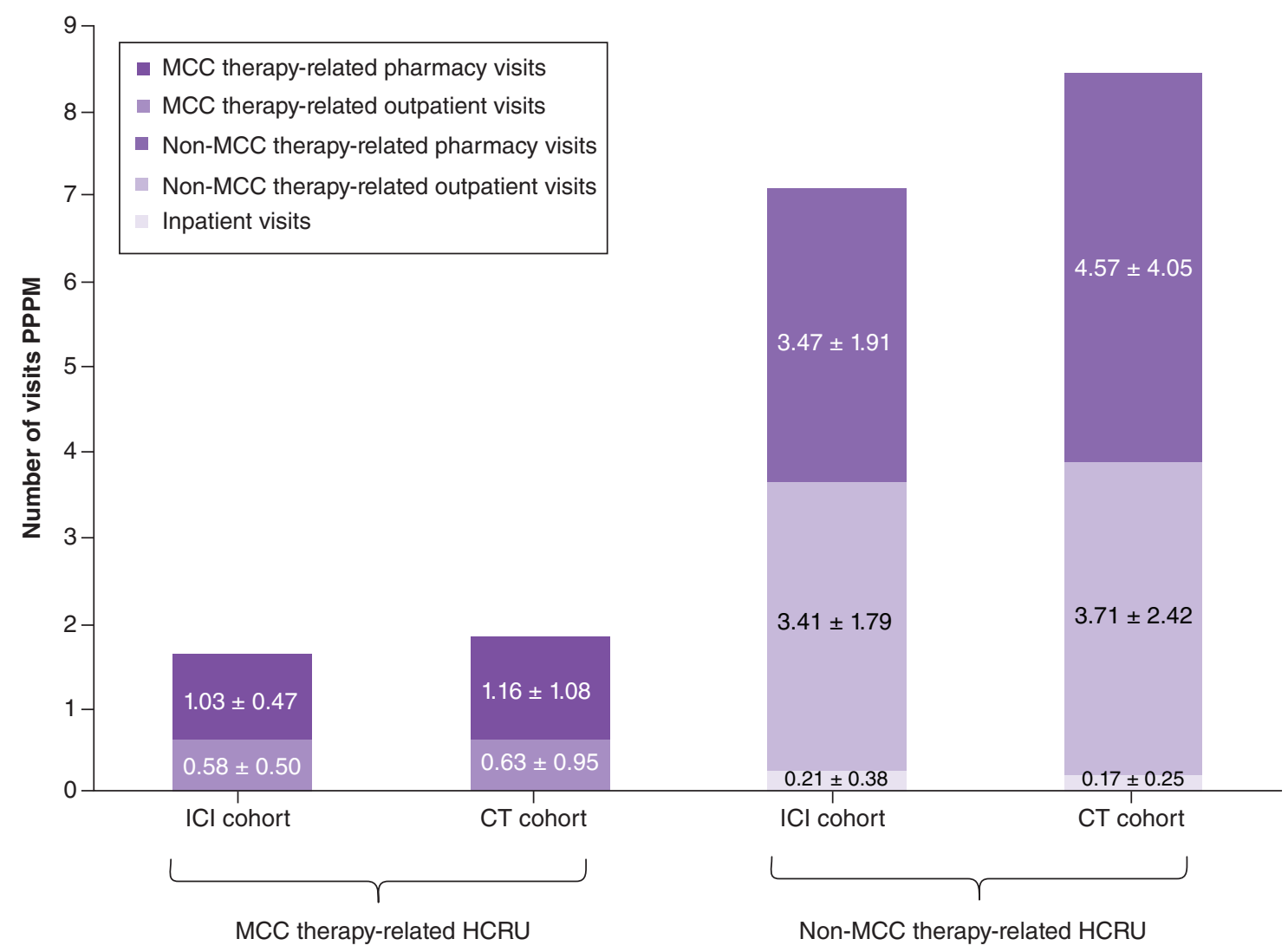

Figure 6. Mean all-cause healthcare resource utilization (per patient per month) among Merkel cell carcinoma patients prescribed recommended first-line systemic therapy during time to next treatment stratified by treatment type. Values are presented as mean \pm standard deviation.

CT: Chemotherapy; HCRU: Healthcare resource utilization; ICI: Immune checkpoint inhibitor; MCC: Merkel cell carcinoma; PPPM: Per patient per month.

of PFS, a measure of continued clinical benefits, that has been reported in clinical trials [53]. Therefore, our results suggest more durable clinical benefits with ICIs compared with CTs. It should be noted that median time to death was not achieved for the ICI cohort in this current study; this area should be explored in future studies with larger samples sizes and longer follow-up periods. However, the median time to death for the CT cohort in this study ( $\sim 13$ months) is higher than the estimates in previous research which ranged from 9.5 to 12 months [24,40,54]. This discrepancy may be reflective of the restriction in our study to only CTs recommended by the NCCN.

Very few real-world studies have explored HCRU and costs in MCC patients; to our knowledge, none have been performed to consider the economic burden associated with ICIs. Previous studies focused on the treatment patterns and economic burden among MCC patients treated with traditional treatment regimens (i.e, radiotherapy, CT and surgery). In a study conducted by Steuten $e$ al. [40], the treatment patterns, overall survival and annual direct healthcare costs were measured for patients with advanced MCC using Surveillance, Epidemiology, and End Result-Medicare data from 2006 to 2013. In this study, total 12-month direct healthcare costs were highest among patients undergoing CT alone or in combination with other therapies. Kearney et al. estimated mean annual costs among patients treated with CT alone in different lines to be $\$ 194,463 \pm \$ 382,463$ [19], much higher than the PPPY total cost estimate for the CT cohort in this study $(\$ 131,482 \pm \$ 165,908)$; the discrepancy between the estimates in both studies may be due to difference in study design and observation periods for costs (i.e., TTNT in our study vs duration of $1 \mathrm{~L}$ therapy in the study by Kearney et al.). Our study also suggests that patients treated with ICIs incur higher total costs, driven by higher outpatient and pharmacy costs, than patients treated with CTs. This might be reflective of the higher costs of acquiring and administering expensive ICI drugs [19]; for example, 


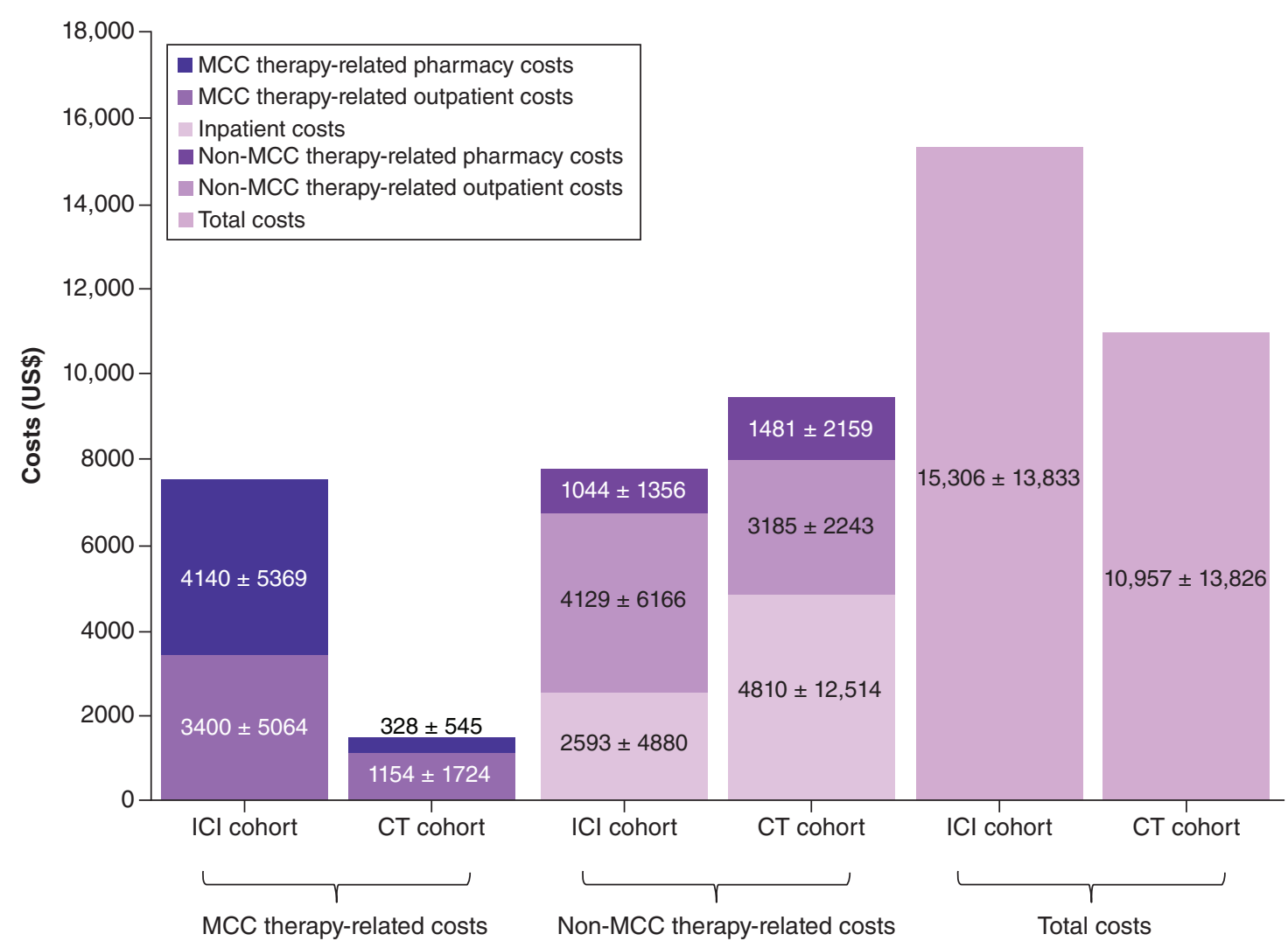

Figure 7. Mean all-cause healthcare costs (per patient per month) among Merkel cell carcinoma patients prescribed recommended first-line systemic therapy during time to next treatment stratified by treatment type. Values are presented as mean \pm standard deviation.

CT: Chemotherapy; ICl: Immune checkpoint inhibitor; MCC: Merkel cell carcinoma.

49.3 and $13.5 \%$ of the costs were MCC therapy-related for the ICI and CT cohorts, respectively. On the other hand, non-MCC therapy-related costs were $50.7 \%$ of total costs ( $43.9 \%$ due to medical services) for the ICI cohort and $86.5 \%$ (73.0\% due to medical services) for the CT cohort; this is also characterized by lower inpatient costs for the ICI cohort $(\$ 2593 \pm \$ 4880)$ than the CT cohort $(\$ 4810 \pm \$ 12,514)$. This, together with the shorter length of inpatient stay for the ICI cohort, suggests a lower burden due to inpatient services for the ICI cohort, despite higher MCC therapy-related and total costs. To validate these findings, future research should compare economic burden between MCC patients treated with ICI therapy versus CT in the $1 \mathrm{~L}$ setting using appropriate statistical methodology to adjust for baseline characteristics and test for significance.

Although a compromised immune system is an established risk factor for MCC, only $24.3 \%$ of the patients were identified as immunocompromised in this study [56,57]. This may be due to differences between the criteria for identifying immunocompromised patients in clinical practice, where the designation is not clear-cut and based on physician's opinion, and the criteria used in our study, which is based on a comprehensive algorithm [58]. However, our results are in alignment with a previous study, which used the same comprehensive algorithm for the identification of immunocompromised patients, and in which $23.9 \%$ of mMCC patients were found to be immunocompromised [58]. This demonstrates the reliability of these criteria. In addition, these estimates are higher than what have been previously reported [59]; this could be due to the inclusion of more proxies in the definition used by Cowey et al. [58], and results should be interpreted accordingly. Future studies using data which include electronic medical records, in which immunocompromised status is clearly indicated, are needed to validate our findings. Nonetheless, our study suggests a numerically higher baseline clinical and economic burden among immunocompromised patients with newly diagnosed MCC compared with their nonimmunocompromised counterparts. This is in alignment with the criteria for immunocompromised status used in this study which was based on diagnoses or 
Table 2. Baseline demographic and clinical characteristics of Merkel cell carcinoma patients prescribed first-line systemic therapy stratified by immunocompromised status.

Demographic and clinical characteristics

MCC patients with $1 \mathrm{~L}$ systemic therapy $(n=74)$

\begin{tabular}{|c|c|c|}
\hline & Immunocompromised cohort $(n=18)$ & Nonimmunocompromised cohort $(n=56)$ \\
\hline Age (years; mean $\pm S D$, median [range]) & $70.5 \pm 8.5,68.0(55.0-84.0)$ & $69.8 \pm 7.3,69.0(50.0-85.0)$ \\
\hline \multicolumn{3}{|l|}{ Age category $(n, \%)$} \\
\hline$-\geq 65$ years & $13(72.2 \%)$ & $48(85.7 \%)$ \\
\hline \multicolumn{3}{|l|}{ Gender (n, \%) } \\
\hline - Male & $18(100 \%)$ & $55(98.2 \%)$ \\
\hline \multicolumn{3}{|l|}{ Race $(n, \%)$} \\
\hline - White & $15(83.3 \%)$ & $50(89.3 \%)$ \\
\hline \multicolumn{3}{|l|}{ Baseline clinical characteristics } \\
\hline - Quan-CCl score (mean \pm SD) & $8.6 \pm 4.1,9.0(0.0-15.0)$ & $5.8 \pm 4.0,6.0(0.0-15.0)$ \\
\hline \multicolumn{3}{|l|}{ Baseline comorbidities ( $\mathrm{n}, \%$ ) } \\
\hline \multicolumn{3}{|l|}{$\mathrm{CCl}$ conditions } \\
\hline - Any malignancy & $10(55.6 \%)$ & $31(55.4 \%)$ \\
\hline - Metastatic solid tumor & $15(83.3 \%)$ & $31(55.4 \%)$ \\
\hline - Other & $12(66.7 \%)$ & $37(66.1 \%)$ \\
\hline Time from MCC date to index date (days; mean $\pm S D$, median [range]) & $173 \pm 146,146(2-493)$ & $122 \pm 139,56(0-538)$ \\
\hline \multicolumn{3}{|l|}{ Baseline economic measures (mean \pm SD, median [range]) } \\
\hline \multicolumn{3}{|l|}{ Baseline all-cause HCRU PPPM } \\
\hline - Inpatient visits & $0.27 \pm 0.34,0.17(0.00-1.33)$ & $0.13 \pm 0.25,0(0.00-1.67)$ \\
\hline - Outpatient visits & $5.26 \pm 2.56,4.92(1.50-11.0)$ & $3.93 \pm 2.51,3.42(0.50-12.83)$ \\
\hline - Pharmacy visits & $5.43 \pm 3.10,4.08(1.83-12.17)$ & $3.38 \pm 2.76,2.83(0.00-15.00)$ \\
\hline \multicolumn{3}{|l|}{ Baseline MCC-related HCRU PPPM } \\
\hline - Inpatient visits & $0.15 \pm 0.18,0.17(0.00-0.67)$ & $0.06 \pm 0.11,0(0.00-0.50)$ \\
\hline - Outpatient visits & $1.08 \pm 1.02,0.83(0.00-3.50)$ & $1.15 \pm 1.50,0.67(0.00-7.00)$ \\
\hline \multicolumn{3}{|l|}{ Baseline all-cause healthcare costs PPPM } \\
\hline - Inpatient costs & $\$ 5262 \pm \$ 8902, \$ 1769(\$ 0-\$ 36,296)$ & $\$ 3173 \pm \$ 5937, \$ 0(\$ 0-\$ 34,924)$ \\
\hline - Outpatient costs & $\$ 4059 \pm \$ 2456, \$ 3525(\$ 784-\$ 10,485)$ & $\$ 3557 \pm \$ 2821, \$ 3024(\$ 310-\$ 13,799)$ \\
\hline - Pharmacy costs & $\$ 784 \pm \$ 1101, \$ 406(\$ 16-\$ 4495)$ & $\$ 426 \pm \$ 1093, \$ 129(\$ 0-\$ 7836)$ \\
\hline - Total medical costs (outpatient + inpatient) & $\$ 9320 \pm \$ 10,626, \$ 5666(\$ 2478-\$ 46,782)$ & $\$ 6730 \pm \$ 7345, \$ 3651(\$ 310-\$ 40,568)$ \\
\hline - Total costs (outpatient + inpatient + pharmacy) & $\$ 10,104 \pm \$ 10,702, \$ 6647(\$ 2729-\$ 47,580)$ & $\$ 7156+\$ 7634, \$ 3774(\$ 319-\$ 41,866)$ \\
\hline \multicolumn{3}{|l|}{ Baseline MCC-related healthcare costs PPPM } \\
\hline - Inpatient costs & $\$ 2249 \pm \$ 3705, \$ 829(\$ 0-\$ 15,056)$ & $\$ 1590 \pm \$ 3456, \$ 0(\$ 0-\$ 13,846)$ \\
\hline - Outpatient costs & $\$ 1292 \pm \$ 1132, \$ 1282(\$ 0-\$ 3881)$ & $\$ 1358 \pm \$ 2023, \$ 613(\$ 0-\$ 10,339)$ \\
\hline - Total medical costs (outpatient + inpatient) & $\$ 3541 \pm \$ 3357, \$ 2733(\$ 238-\$ 15,056)$ & $\$ 2949 \pm \$ 4217, \$ 899(\$ 0-\$ 14,933)$ \\
\hline
\end{tabular}

treatments for serious comorbidities. We did not pursue further analyses with the immunocompromised population due to small sample size and reporting restrictions under the VHA data user agreement. Therefore, we suggest this area for future large scale studies to explore. For example, future studies can test the hypothesis that clinical and economic outcomes may be worse for immunocompromised than nonimmunocompromised mMCC patients, due to the inability of the former to mount strong immune responses to tumors.

This study is subject to the limitations inherently associated with claims-based studies including coding errors, insufficient/missing information and the subsequent use of algorithms as proxies for study measures. Also, our study findings may only be applicable to VHA-enrolled patients (i.e., older, male and economically disadvantaged) diagnosed with MCC. Thus, the results of our study may not be generalizable to the US cancer population. However, the VHA is the largest integrated healthcare system in the USA and a major cancer care provider, and it is worth noting that the findings in this study are consistent with those from previous research using other data sources. In addition, the small sample size did not permit us to report more granular information on our patient populations due to reporting limitations associated with using VHA data. Small sample size may have 
also limited our ability to more accurately quantify patient characteristics and outcome measures for the ICI cohort. Comparative analysis between the cohorts was not performed due to sample size constraints and the small number of outcome events observed. Therefore, determination of the relationship between study outcomes and treatment types is an early signal/trend rather than being conclusive. The use of NCCN-recommended regimens as a proxy for metastatic disease did not consider the use of chemotherapy as adjuvant; however, chemotherapy is not generally recommended for the treatment of earlier stages of MCC and the use of adjuvant chemotherapy is rare [21]. Nonetheless, the results warrant future studies that compare outcomes between patients with MCC treated with ICI versus chemotherapy after excluding any possible use of chemotherapy as adjuvant. Finally, the study population was composed of mostly elderly patients who, being eligible, may also be enrolled with Medicare. Healthcare services covered by Medicare are not accounted for in the VHA database and this may have led to an underreporting of HCRU and underestimation of healthcare costs.

\section{Conclusion}

In summary, this noncomparative, claims-based study suggests that the 1L use of ICIs is associated with longer treatment duration and TTNT than NCCN-recommended CTs among VHA-enrolled patients with MCC in the real-world setting. This adds to existing evidence building on the advantages of ICI therapies in the management of MCC. However, the findings of this study warrant investigation in future studies with larger populations.

\section{Summary points}

- Historically, chemotherapy (CT) has been used to treat metastatic Merkel cell carcinoma (mMCC) but is associated with short duration of response and high toxicity, especially among elderly patients.

- Clinical trials with immune checkpoint inhibitors (ICls) have shown promising results among patients mMCC and led to the approval of two $\mathrm{ICl}$ agents for the management of this malignancy: avelumab and pembrolizumab.

- The purpose of this study was to examine patient characteristics, treatment duration and clinical and economic outcomes among treatment-naive MCC patients treated with $\mathrm{ICl}$ and NCCN-recommended CT therapies.

- Patients with newly diagnosed MCC from the Veterans Health Administration database from 1 October 2013 to 21 January 2018 were assigned, in parallel, to cohorts based on the type of first-line treatment (ICI or CT) and immunocompromised status (yes/no).

- Patient demographic and baseline clinical characteristics were obtained for each cohort while time to death, time to next treatment (TTNT), healthcare resource utilization and costs during TTNT and treatment duration (obtained from Kaplan-Meier analysis) were computed for the treatment cohorts only.

- The demographic profile of each cohort was mostly White, male and elderly and with substantial clinical comorbidity.

- Median duration of therapy was 300 days (95\% Cl: 91-364 days) for ICls and 91 days (95\% Cl: 63-105 days) for CTs. The median TTNT was 245 (95\% Cl: 124 days - upper limit not reached) and 184 days (95\% Cl: 127-324 days), respectively. Median time to death was not reached for the ICl cohort ( $95 \% \mathrm{Cl}: 132$ days - upper limit not reached) but was 403 days ( $95 \% \mathrm{Cl}$ : 280-630 days) for the CT cohort.

- During TTNT, the ICl cohort incurred mean ( \pm standard deviation [SD]) all-cause per patient per month (PPPM) direct healthcare costs of $\$ 15,306 \pm \$ 13,833$, of which $\$ 7766 \pm \$ 7744$ were due to non-MCC therapy-related services, while the CT cohort incurred mean all-cause PPPM direct healthcare costs of $\$ 10,957 \pm \$ 13,826$, of which $\$ 9476 \pm \$ 13,598$ were due to non-MCC therapy-related services.

- Our study suggests a numerically higher mean $( \pm S D)$ baseline clinical burden among immunocompromised patients with newly diagnosed MCC compared with their nonimmunocompromised counterparts (Quan-Charlson Comorbidity Index score, immunocompromised cohort: $8.6 \pm 4.1$; nonimmunocompromised cohort: $5.8 \pm 4.0$ ). Not surprisingly, the baseline mean all-cause PPPM total healthcare costs were higher for the immunocompromised than the nonimmunocompromised cohort, at $\$ 10,104 \pm \$ 10,702$ and $\$ 7156 \pm \$ 7634$, respectively.

- This retrospective real-world study suggests initial clinical benefits associated with ICI therapies compared with CT therapies in the first-line MCC setting that need to be confirmed in future large-scale studies using appropriate comparative statistical techniques. 
Financial \& competing interests disclosure

This study was sponsored by EMD Serono, Inc. (http://dx.doi.org/10.13039/100009945); a business of Merck KGaA, Darmstadt, Germany, It is part of an alliance between EMD Serono, Inc. and Pfizer, Inc. (http://dx.doi.org/10.13039/100004319). S Chandra is an employee of Northwestern Medicine; M Kearney is an employee of Merck KGaA. Y Zheng, T Yu and H Phatak are employees of EMD Serono, Inc. (a business of Merck KGaA). S Pandya and L Wang are employees of STATinMED Research, which is a paid consultant to Pfizer, Inc. and EMD Serono, Inc. R Kim is an employee of Pfizer, Inc. Data from this study were previously presented in poster format at ASCO-SITC Clinical Immune-Oncology Symposium 2019 and as an abstract at the 2019 ASCO Annual Meeting. The authors have no other relevant affiliations or financial involvement with any organization or entity with a financial interest in or financial conflict with the subject matter or materials discussed in the manuscript apart from those disclosed.

Statistical programming and medical writing assistance were provided by $Y$ Cao and C Dieyi of STATinMED Research, respectively. Editorial support was provided by M Moriarty, also of STATinMED Research.

Ethical conduct of research

Since the core study described herein did not involve the collection, use or transmittal of individual identifiable data, IRB approval to conduct this study was not required. The security of the data meets the requirements of the Health Insurance Portability and Accountability Act (HIPAA) of 1996, and this study adheres to the principles outlined in the Declaration of Helsinki.

\section{Open access}

This work is licensed under the Attribution-NonCommercial-NoDerivatives 4.0 Unported License. To view a copy of this license, visit http://creativecommons.org/licenses/by-nc-nd/4.0/

\section{References}

Papers of special note have been highlighted as: • of interest; $\bullet \bullet$ of considerable interest

1. Lemos BD, Storer BE, Iyer JG et al. Pathologic nodal evaluation improves prognostic accuracy in Merkel cell carcinoma: analysis of 5823 cases as the basis of the first consensus staging system. J. Am. Academy Dermatol. 63(5), 751-761 (2010).

2. Heath M, Jaimes N, Lemos B, Mostaghimi A, Wang LC, Penas PF. Clinical characteristics of Merkel cell carcinoma at diagnosis in 195 patients: the AEIOU features. J. Am. Acad. Dermatol. 58, 375-381 (2008).

3. Saini AT, Miles BA. Merkel cell carcinoma of the head and neck: pathogenesis, current and emerging treatment options. OncoTargets Ther. 8, 2157-2167 (2015).

4. Chen MM, Roman SA, Sosa JA et al. The role of adjuvant therapy in the management of head and neck Merkel cell carcinoma: an analysis of 4815 patients. JAMA Otolaryngol. Head Neck Surg. 141, 137-141 (2015).

5. Smith VA, Camp ER, Lentsch EJ. Merkel cell carcinoma: identification of prognostic factors unique to tumors located in the head and neck based on analysis of SEER data. Laryngoscope 122, 1283-1290 (2012).

6. Duprat JP, Landman G, Salvajoli JV, Brechtbühl ER. A review of the epidemiology and treatment of Merkel cell carcinoma. Clinics 66(10), 1817-1823 (2011).

7. Paulson KG, Park SY, Vandeven NA et al. Merkel cell carcinoma: current US incidence and projected increases based on changing demographics.. J. Am. Acad. Dermatol. 78(3), 457-463.e2 (2018).

8. Fitzgerald TL, Dennis S, Kachare SD, Vohra NA, Wong JH, Zervos EE. Dramatic increase in the incidence and mortality from Merkel cell carcinoma in the United States. Am. Surgeon 81(8), 802-806 (2015).

9. Liang E, Brower JV, Rice SR, Buehler DG, Saha S, Kimple RJ. Merkel cell carcinoma analysis of outcomes: a 30-year experience. PLoS ONE 10(6), e0129476 (2015).

10. Feng H, Shuda M, Chang Y, Moore PS. Clonal integration of a polyomavirus in human Merkel cell carcinoma. Science 319, 1096-1100 (2008).

11. Goh G, Walradt T, Markarov V, et al. Mutational landscape of MCPyV-positive and MCPyV-negative Merkel cell carcinomas with implications for immunotherapy. Oncotarget 7(3), 3403 (2016).

12. Chan TA, Nghiem P, Lifton RP et al. Mutational landscape of MCPyV-positive and MCPyV-negative Merkel cell carcinomas with implications for immunotherapy. Oncotarget 7, 3403-3415 (2016).

13. Wong SQ, Waldeck K, Vergara IA et al. UV-associated mutations underlie the etiology of MCV-negative Merkel cell carcinomas. Cancer Res. 75, 5228-5234 (2015).

14. Harms PW, Vats P, Verhaegen ME et al. The distinctive mutational spectra of polyomavirus-negative Merkel cell carcinoma. Cancer Res. 75, 3720-3727 (2015).

15. Becker JC. Merkel cell carcinoma. Ann. Oncol. 21(Suppl. 7), vii81-85 (2010).

16. Cornejo C, Miller CJ. Merkel cell carcinoma: updates on staging and management. Dermatol. Clin. 37(3), 269-277 (2019). 
17. Femia D, Prinzi N, Anichini A et al. Treatment of advanced Merkel cell carcinoma: current therapeutic options and novel immunotherapy approaches. Target Oncol. 13(5), 567-582 (2018).

18. Villani A, Fabbrocini G, Costa C, Annunziata MC, Scalvenzi M. Merkel cell carcinoma: therapeutic update and emerging therapies. Dermatol Ther. 9(2), 209-222 (2019).

19. Kearney M, Thokagevistk K, Boutmy E, Bharmal M. Treatment patterns, comorbidities, healthcare resource use, and associated costs by line of chemotherapy and level of comorbidity in patients with newly-diagnosed Merkel cell carcinoma in the United States. J. Med. Econ. 21(12), 1159-1171 (2018).

- Real-world study shows that the costs associated with chemotherapy were higher than costs associated with surgery, radiotherapy or combinations of these therapies among newly diagnosed treatment-naive Merkel cell carcinoma (MCC) patients, underscoring the need for newer therapy options.

20. National Comprehensive Cancer Network. NCCN Clinical Practice Guidelines in Oncology (NCCN guidelines ${ }^{\circledR}$ ): Merkel cell carcinoma V1.2018. (2018). https://www.nccn.org/professionals/physician_gls/pdf/mcc.pdf

21. Beebe V. A review of Merkel cell carcinoma for dermatology nurses. J. Dermatol. Nurs. Association 1(3), 173-179 (2009).

22. Ghadjar P, Kaanders JH, Poortmans $\mathrm{P}$ et al. The essential role of radiotherapy in the treatment of Merkel cell carcinoma: a study from the rare cancer network. Int. J. Radiat. Oncol. Biol. Phys. 81(4), e583-591 (2011).

23. Lebbe C, Becker JC, Grob JJ et al. Diagnosis and treatment of Merkel cell carcinoma. European consensus-based interdisciplinary guideline. Eur. J. Cancer 51, 2396-2403 (2015).

24. Iyer JG, Blom A, Doumani R et al. Response rates and durability of chemotherapy among 62 patients with metastatic Merkel cell carcinoma. Cancer Med. 5(9), 2294-2301 (2016).

25. Tai PT, Yu E, Winquist E et al. Chemotherapy in neuroendocrine/Merkel cell carcinoma of the skin: case series and review of 204 cases. J. Clin. Oncol. 18, 2493-2499 (2000).

26. Satpute SR, Ammakkanavar NR, Einhorn LH. Role of platinum-based chemotherapy for Merkel cell tumor in adjuvant and metastatic settings. J. Clin. Oncol. 32, 9049 (2014).

27. Chan IS, Bhatia S, Kaufman HL, Lipson EJ. Immunotherapy for Merkel cell carcinoma: a turning point in patient care. J. Immunother. Cancer 6(1), 23 (2018).

28. Garcia-Carbonero R, Marquez-Rodas I, de la Cruz-Merino L et al. Recent therapeutic advances and change in treatment paradigm of patients with Merkel cell carcinoma. Oncologist 24(10), 1375-1383 (2019).

29. Samimi M. Immune checkpoint inhibitors and beyond: an overview of immune-based therapies in Merkel cell carcinoma. Am. J. Clin. Dermatol. 19, 1-7 (2019).

30. Kaufman HL, Russell J, Hamid O et al. Avelumab in patients with chemotherapy-refractory metastatic Merkel cell carcinoma: a multicentre, single-group, open-label, phase 2 trial. Lancet Oncol. 17(10), 1374-1385 (2016).

- JAVELIN Merkel 200 Phase II clinical trial shows that second-line avelumab treatment of MCC was associated with sustained responses and manageable safety profiles in North America, Europe, Australia and Asia.

31. Kaufman HL, Russell JS, Hamid O et al. Updated efficacy of avelumab in patients with previously treated metastatic Merkel cell carcinoma after $\geq 1$ year of follow-up: JAVELIN Merkel 200, a phase 2 clinical trial. J. Immunother. Cancer 6(1), 7 (2018).

-• Updated JAVELIN Merkel 200 Phase II clinical trial shows that with longer follow-up times, chemotherapy-refractory patients with MCC continued to respond to avelumab and showed prolonged progression-free survival.

32. Nghiem P, Bhatia S, Lipson EJ et al. Durable tumor regression and overall survival in patients with advanced merkel cell carcinoma receiving pembrolizumab as first-line therapy. J. Clin. Oncol. 37(9), 693-702 (2019).

- Cancer Immunotherapy Trials Network protocol 9/Keynote-017 Phase II clinical trial shows durable antitumor activity and survival among treatment-naive MCC patients treated with pembrolizumab.

33. Palla AR, Doll D. Immunotherapy in Merkel cell carcinoma: role of avelumab. Immunotargets Ther. 7, 15 (2018).

34. Zanetti I, Coati I, Alaibac M. Interaction between Merkel cell carcinoma and the immune system: pathogenetic and therapeutic implications. Mol. Clin. Oncol. 7(5), 729-732 (2017).

35. Becker JC. Merkel cell carcinoma. Ann. Oncol. 21(Suppl. 7), vii81-85 (2010).

36. Paulson KG, Iyer JG, Blom A et al. Systemic immune suppression predicts diminished Merkel cell carcinoma-specific survival independent of stage. J. Invest. Dermatol. 133(3), 642-646 (2013).

37. Paulson KG, Iyer JG, Simonson WT et al. CD8 ${ }^{+}$lymphocyte intratumoral infiltration as a stage-independent predictor of Merkel cell carcinoma survival: a population-based study. Am. J. Clin. Pathol. 142(4), 452-458 (2014).

38. Sihto H, Böhling T, Kavola $\mathrm{H}$ et al. Tumor infiltrating immune cells and outcome of Merkel cell carcinoma: a population-based study. Clin. Cancer Res. 18(10), 2872-2881 (2012).

39. Behr DS, Peitsch WK, Hametner C et al. Prognostic value of immune cell infiltration, tertiary lymphoid structures and PD-L1 expression in Merkel cell carcinomas. Int. J. Clin. Exp. Pathol. 7(11), 7610 (2014). 
40. Steuten L, Garmo V, Phatak H et al. Treatment patterns, overall survival, and total healthcare costs of advanced Merkel cell carcinoma in the USA. Appl. Health Econ. Health Policy 17(5), 733-740 (2019).

-• Real-world claims-based study analyzes overall survival and healthcare costs among treatment-naive MCC patients treated with surgery, radiation and/or chemotherapy alone or in combination.

41. Kearney M, Esposito DB, Peñalvo JL et al. Economic burden of locally advanced or metastatic Merkel cell carcinoma in the United States: an analysis of electronic health records. Value Health 21(1), S45 (2018).

42. Riemenschneider K, Liu J, Power JG. Skin cancer in the military: a systematic review of melanoma and nonmelanoma skin cancer incidence, prevalence and screening among active duty and veteran personnel. J. Am. Acad. Dermatol. 78(6), 1185-1192 (2018).

43. Stokes JB, Graw KS, Dengel LT et al. Patients with Merkel cell carcinoma tumors $\leq 1.0 \mathrm{~cm}$ in diameter are unlikely to harbor regional lymph node metastasis. J. Cin. Oncol. 27(23), 3772-3777 (2009).

44. Quigley PA, Palacios P, Spehar AM. Veterans' fall risk profile: a prevalence study. Clin. Interv. Aging 1(2), 169 (2006).

45. Bagalman E. The number of veterans that use VA health care services: a fact sheet. https://fas.org/sgp/crs/misc/R 43579.pdf

46. Baser O, Xie L, Pesa J, Durkin M. Healthcare utilization and costs of Veterans Health Administration patients with schizophrenia treated with paliperidone palmitate long-acting injection or oral atypical antipsychotics. J. Med. Econ. 18, 357-365 (2015).

47. Quan $\mathrm{H}$, Sundararajan V, Halfon P et al. Coding algorithms for defining comorbidities in ICD-9-CM and ICD-10 administrative data. Med. Care 43(11), 1130-1192 (2005).

48. Tung A, Hepp Z, Bansal A et al. Characterizing health care utilization, direct costs, and comorbidities associated with interstitial cystitis: a retrospective claims analysis. J. Manag. Care Spec. Pharm. 23(4), 474-482 (2017).

49. US Bureau of Labor Statistics Consumer price index. (2001) http://www.bls.gov/cpi/

50. Allen PJ, Zhang ZF, Coit DG. Surgical management of Merkel cell carcinoma. Ann. Surg. 229(1), 97 (1999).

51. Allen PJ, Bowne WB, Jaques DP, Brennan MF, Busam K, Coit DG. Merkel cell carcinoma: prognosis and treatment of patients from a single institution. J. Clin. Oncol. 23(10), 2300-2309 (2005).

52. Andea AA, Coit DG, Amin B, Busam KJ. Merkel cell carcinoma: histologic features and prognosis. Cancer 113(9), 2549-2558 (2008).

53. D’Angelo SP, Russell J, Lebbé C et al. Efficacy and safety of first-line avelumab treatment in patients with stage IV metastatic Merkel cell carcinoma: a preplanned interim analysis of a clinical trial. JAMA Oncol. 4(9), e180077 (2018).

- JAVELIN Merkel 200 Phase II clinical trial part B shows impressive and durable antitumor activity and manageable safety profiles among treatment-naive MCC patients treated with avelumab.

54. Nghiem PT, Bhatia S, Lipson EJ et al. PD-1 blockade with pembrolizumab in advanced Merkel-cell carcinoma. New Engl. J. Med. 374(26), 2542-2552 (2016).

55. Topalian SL, Bhatia S, Hollebecque A et al. CT074-Non-comparative, open-label multiple cohort phase $1 / 2$ study to evaluate nivolumab (NIVO) in patients with virus-associated tumors (CheckMate 358): efficacy and safety in Merkel cell carcinoma (MCC). Proceedings of the American Association for Cancer Research Annual Meeting 2017; Apr 1-5; Washington, DC. Philadelphia (PA): AACR. Cancer Res. 77(Suppl. 13), CT074 (2017).

56. Hughes MP, Hardee ME, Cornelius LA, Hutchins LF, Becker JC, Gao L. Merkel cell carcinoma: epidemiology, target, and therapy. Curr. Dermatol. Rep. 3(1), 46-53 (2014).

57. Schadendorf D, Lebbé C, Zur Hausen A et al. Merkel cell carcinoma: epidemiology, prognosis, therapy and unmet medical needs. Eur. J. Cancer 71, 53-69 (2017).

58. Cowey CL, Mahnke L, Espirito J, Helwig C, Oksen D, Bharmal M. Real-world treatment outcomes in patients with metastatic Merkel cell carcinoma treated with chemotherapy in the USA. Future Oncol. 13(19), 1699-1710 (2017).

-• An observational study which uses the same criteria as used in the current study for defining immunocompromised MCC patients.

59. Heath M, Jaimes N, Lemos B, et al. Clinical characteristics of Merkel cell carcinoma at diagnosis in 195 patients: the AEIOU features. J. Am. Acad. Dermatol. 58(3), 375-381 (2008). 